\title{
The Complexity of Definability by Open First-Order Formulas
}

\author{
Carlos Areces \\ carlos.areces@gmail.com
}

\author{
Daniel Penazzi \\ danielpenazzi@gmail.com
}

Miguel Campercholi

mcampercholi@gmail.com

Pablo Ventura

pablogventura@gmail.com

\section{Universidad Nacional de Córdoba and CONICET \\ Córdoba, Argentina}

\begin{abstract}
In this article we formally define and investigate the computational complexity of the Definability Problem for open first-order formulas (i.e., quantifier free first-order formulas) with equality. Given a logic $\mathcal{L}$, the $\mathcal{L}$-Definability Problem for finite structures takes as input a finite structure $\boldsymbol{A}$ and a target relation $T$ over the domain of $\boldsymbol{A}$, and determines whether there is a formula of $\mathcal{L}$ whose interpretation in $\boldsymbol{A}$ coincides with $T$. We show that the complexity of this problem for open first-order formulas (open definability, for short) is coNPcomplete. We also investigate the parametric complexity of the problem, and prove that if the size and the arity of the target relation $T$ are taken as parameters then open definability is coW[1]-complete for every vocabulary $\tau$ with at least one, at least binary, relation.
\end{abstract}

\section{Introduction}

Arguably, any attempt to provide a logic $\mathcal{L}$ with a formal semantics starts with the definition of a function that, given a suitable structure $\boldsymbol{A}$ for $\mathcal{L}$ and 
a formula $\varphi$ in $\mathcal{L}$, returns the extension of $\varphi$ in $\boldsymbol{A}$. Usually, this extension is a set of tuples built from elements in $\boldsymbol{A}$. These extensions, also called definable sets, are the elements that will be referred by the formulas of $\mathcal{L}$ in a given structure, and in that sense, define the expressivity of $\mathcal{L}$. The definable sets of $\boldsymbol{A}$ are the only objects that $\mathcal{L}$ can see. For that reason, definable sets are one of the central objects studied by Model Theory. It is usually an interesting question to investigate, given a logic $\mathcal{L}$, which are the definable sets of $\mathcal{L}$ over a given structure $\boldsymbol{A}$, or, more concretely, whether a particular set of tuples is a definable set of $\mathcal{L}$ over $\boldsymbol{A}$. This is what we call the Definability Problem for $\mathcal{L}$ over $\boldsymbol{A}$.

In this article we investigate the computational complexity of the definability problem for open first-order formulas -i.e., quantifier free first-order formulas- with equality over a relational vocabulary (open-definability, for short).

One of the main goals of Computational Logic is to understand the computational complexity of different problems for different logics. Classically, one of the most investigated inference problems is Satisfiability (SAT, for short): given a formula $\varphi$ from a given logic $\mathcal{L}$ decide whether there exists a structure that makes $\varphi$ true. In recent years, and motivated by concrete applications, other reasoning problems have sparkled interest. A well known example is the Model Checking Problem (MC, for short) used in software verification to check that a given property $P$ (expressed as a formula in the verification language) holds in a given formal representation $S$ of the system (see, e.g., [7, 5]). From a more general perspective, MC can be defined as follows: given a structure $\boldsymbol{A}$, and a formula $\varphi$ decide which is the extension $T$ of $\varphi$ in $\boldsymbol{A}$. From that perspective, the definability problem can be understood as the inverse problem of MC: given a structure $\boldsymbol{A}$ and a target set $T$ it asks whether there is a formula $\varphi$ whose extension is $T$. A further example of a reasoning task related to definability comes from a seemingly unrelated field: computational linguistics, more specifically, in the subarea of automated language generation called Generation of Referring Expressions (GRE). The GRE problem can be intuitively understood as follows: given a context $C$ and an target object $t$ in $C$, generate a grammatically correct description (in some natural language) that represents $t$, differentiating it from other objects in $C$, or report failure if such a description does not exist (see [17] for a survey on GRE). Most of the work in this area is focused on the content determination problem (i.e., finding the properties that singles out the target object) and leaves the actual realization (i.e., expressing this 
content as a grammatically correct expression) to standard techniques. As it is discussed in [2, 1] the content realization part of the GRE problem can be understood as the task that, given a structure $\boldsymbol{A}$ that represents the context $C$, and an object $t$ in the domain of $\boldsymbol{A}$ returns a formula $\varphi$ in a suitable logic $\mathcal{L}$ whose extension in $\boldsymbol{A}$ coincides with $t$. Of course, this will be possible only if $t$ is definable for $\mathcal{L}$ over $\boldsymbol{A}$.

The complexity of the definability problem for a number of logics has already been investigated. Let FO be first-order logic with equality in a vocabulary without constant symbols. The computational complexity of FOdefinability was already discussed in 1978 [18, 4], when a semantic characterization of the problem, based on automorphisms, placed FO-definability within coNP. Much more recently, in [3], a polynomial-time algorithm for FO-definability was given, which uses calls to a graph-isomorphism subroutine as an oracle. As a consequence, FO-definability is shown to be inside GI (defined as the set of all languages that are polynomial-time Turing reducible to the graph isomorphism problem). The authors also show that the problem is GI-hard and, hence, GI-complete. Interestingly, Willard showed in [19], that the complexity of the definability problem for the fragment of $\mathbf{F O}$ restricted to conjunctive queries (i.e., formulas of the form $\exists \bar{x} \bigwedge_{i} C_{i}$, where each conjunct $C_{i}$ is atomic) was coNEXPTIME-complete. The complexity upper bound followed from a semantic characterization of CQ-definability in terms of polymorphisms given in [16], while the lower bound is proved by an encoding of a suitable tiling problem. The complexity of definability has been investigated also for some modal languages: 2] shows that for the basic modal logic $K$, the definability problem is tractable (i.e., in $\mathrm{P}$ ); in [1] the result is extended to some fragments of $K$ known as $\mathcal{E} \mathcal{L}$ and $\mathcal{E} \mathcal{L}^{+}$. [12 discusses the length of the shortest formula required to define a given target set, proving that for $\mathcal{L} \in\left\{K, \mathcal{E} \mathcal{L}, \mathcal{E} \mathcal{L}^{+}\right\}$, the lower bound for the length of a definition is exponential in the size of the input structure. More precisely, it is shown that there are structures $G_{1}, G_{2}, \ldots$ such that for every $i$, the size of $G_{i}$ is linear in $i$ but the size of the shortest definition for some element in $G_{i}$ is bounded from below by a function which is exponential on $i$.

The article is structured as follows. After introducing basic notations and definitions in Section 2, we show that open-definability is coNP-complete in Section 3. Section 4 discusses the parameterized complexity of the problem. Finally, in Section 5 we show that the length of the shortest open formula that might be required in a definition cannot be bounded by a polynomial: in some cases, definitions by open formulas need to be exponentially long. 


\section{Preliminaries}

In this section we provide some basic definitions and fix notation. We assume basic knowledge of first-order logic. For a detailed account see, e.g., [11].

We focus on definability by open first-order formulas in a purely relational first-order vocabulary, i.e., without function or constant symbols. For a relation symbol $R$ in a vocabulary $\tau$, let $\operatorname{ar}(R)$ denote the arity of $R$. In what follows, all vocabularies are assumed to be finite and purely relational. We assume that the language contains variables from a countable, infinite set VAR $=\left\{x_{1}, x_{2}, \ldots, x_{n}, \ldots\right\}$. Variables from VAR are the only terms in the language. Atomic formulas are either of the form $v_{i}=v_{j}$ or $R(\bar{v})$, where $v_{i}, v_{j} \in \mathrm{VAR}, \bar{v}$ is a sequence of variables in VAR of length $k$ and $R$ is a relation symbol of arity $k$. Open formulas are Boolean combinations of atomic formulas. We shall often write just formula instead of open formula. We write $\varphi\left(v_{1}, \ldots, v_{k}\right)$ for an open formula $\varphi$ whose variables are included in $\left\{v_{1}, \ldots, v_{k}\right\}$.

Let $\tau$ be a vocabulary. A $\tau$-structure (or model) is a pair $\boldsymbol{A}=\left\langle A,{ }^{\boldsymbol{A}}\right\rangle$ where $A$ is a non-empty set (the domain or universe), and ${ }^{\boldsymbol{A}}$ is an interpretation function that assigns to each $k$-ary relation symbol $R$ in $\tau$ a subset $R^{\boldsymbol{A}}$ of $A^{k}$. If $\boldsymbol{A}$ is a structure we write $A$ for its domain and ${ }^{\boldsymbol{A}}$ for its interpretation function. Given a formula $\varphi\left(v_{1}, \ldots, v_{k}\right)$, and a sequence of elements $\bar{a}=\left\langle a_{1}, \ldots, a_{k}\right\rangle \in A^{k}$ we write $\boldsymbol{A} \models \varphi[\bar{a}]$ if $\varphi$ is true in $\boldsymbol{A}$ under an assignment that maps $v_{i}$ to $a_{i}$.

We say that a subset $T \subseteq A^{k}$ is open-definable in $\boldsymbol{A}$ if there is an open first-order formula $\varphi\left(x_{1}, \ldots, x_{k}\right)$ in the vocabulary of $\boldsymbol{A}$ such that

$$
T=\left\{\bar{a} \in A^{k}: \boldsymbol{A} \models \varphi[\bar{a}]\right\} .
$$

In this article we study the following computational decision problem:

$$
\begin{aligned}
& \text { OpenDef Instance: A finite relational structure } \boldsymbol{A} \text { and a relation } \\
& T \text { over the domain of } \boldsymbol{A} \text {. } \\
& \text { Question: Is } T \text { open-definable in } \boldsymbol{A} \text { ? }
\end{aligned}
$$

Let $f: \operatorname{dom} f \subseteq A \rightarrow A$ be a function. Given $S \subseteq A^{m}$, we say that $f$ preserves $S$ if for all $\left\langle s_{1}, \ldots, s_{m}\right\rangle \in S \cap(\operatorname{dom} f)^{m}$ we have $\left\langle f s_{1}, \ldots, f s_{m}\right\rangle \in S$. The function $f$ is a subisomorphism (subiso for short) of $\boldsymbol{A}$ provided that $f$ 
is injective, and both $f$ and $f^{-1}$ preserve $R^{\boldsymbol{A}}$ for each $R \in \tau$. (Note that a subiso of $\boldsymbol{A}$ is exactly an isomorphism between two substructures of $\boldsymbol{A}$.) We denote the set of all subisomorphisms of $\boldsymbol{A}$ by subIso $\boldsymbol{A}$.

The following semantic characterization of open-definability is central to our study.

Theorem 1 ([․, Thm 3.1]). Let $\boldsymbol{A}$ be a finite relational structure and $T \subseteq$ $A^{m}$. The following are equivalent:

1. $T$ is open-definable in $\boldsymbol{A}$.

2. $T$ is preserved by all subisomorphisms $\gamma$ of $\boldsymbol{A}$.

3. $T$ is preserved by all subisomorphisms $\gamma$ of $\boldsymbol{A}$ with $|\operatorname{dom} \gamma| \leq m$.

Proof. The equivalence of (11) and (2) is proved in [6, Thm 3.1]. Certainly (2) implies (3), so we show that (3) implies (2). Let $\gamma$ be a subiso of $\mathbf{A}$ and let $\left\langle a_{1}, \ldots, a_{m}\right\rangle \in T \cap(\operatorname{dom} \gamma)^{m}$. Note that the restriction $\left.\gamma\right|_{\left\{a_{1}, \ldots, a_{m}\right\}}$ is a subiso of A. Thus, $\gamma(\bar{a})=\left.\gamma\right|_{\left\{a_{1}, \ldots, a_{m}\right\}}(\bar{a}) \in T$.

\subsection{Encodings and Sizes}

As is customary when considering complexity questions, the size of an object is the length of a string over a finite alphabet encoding the object. We assume fixed encodings for vocabularies, relations, structures and formulas, and define the size of these objects according to these encodings. For a set $S$, let $|S|$ be the number of elements in $S$, and for a relational vocabulary $\tau$, let $|\tau|$ be the number of relational symbols in $\tau$.

We write size $(o b)$ to denote the size of an object $o b$. Even though we do not specify the encodings we assume the following equalities throughout this note. Let $\tau$ be a relational vocabulary, $\mathbf{A}$ a $\tau$-structure, $T \subseteq A^{m}$ and $\varphi$ a first-order formula.

- $\operatorname{size}(\tau)=\left(|\tau|+\sum_{R \in \tau} \operatorname{ar}(R)\right) \log |\tau|, 1$

- $\operatorname{size}(\mathbf{A})=\operatorname{size}(\tau)+\left(|A|+\sum_{R \in \tau} \operatorname{ar}(R)\left|R^{\mathbf{A}}\right|\right) \log |A|$,

- $\operatorname{size}(\mathbf{A}, T)=(\operatorname{size}(\mathbf{A})+m|T|) \log |A|$,

- $\operatorname{size}_{\tau}(\varphi)=\operatorname{relcount}(\varphi) \log |\tau|+\operatorname{varcount}(\varphi) \log (\operatorname{var} \#(\varphi))$.

\footnotetext{
${ }^{1}$ When an expression involving $\log x$ does note make sense, read it as $\max \{\log x, 1\}$.
} 
Here $\operatorname{relcount}(\varphi)[\operatorname{varcount}(\varphi)]$ stands for the number of occurrences of relation symbols [variables] in $\varphi$, and $\operatorname{var}^{\#}(\varphi)$ is the number of different variables occurring in $\varphi$. Since a formula $\varphi$ is a $\tau$-formula for every $\tau$ containing the relation symbols in $\varphi$, the encoding of $\varphi$ (and thus its size) depend on which vocabulary we have in mind for $\varphi$. Another assumption we make on the encodings is that determining whether $\bar{a} \in R^{\mathbf{A}}$ can be computed in time $O(\operatorname{size}(\mathbf{A}))$.

\section{Classical Complexity of Open-Definability}

In what follows a graph is a model $\boldsymbol{G}$ of the vocabulary $\tau_{\mathrm{GRAPH}}=\{E\}$, with $E$ binary, and such that $E^{G}$ is symmetric and irreflexive. We provide a reduction from the following problem to prove our hardness result.

\begin{tabular}{|ll|}
\hline InducedPath & A finite graph $\boldsymbol{G}$ and a positive integer \\
& $k$.
\end{tabular} \mid $\begin{aligned} & \text { Does } \boldsymbol{G} \text { have a path of length } k \text { as an } \\
& \text { induced subgraph (i.e., as a submodel)? }\end{aligned}$

InducedPath is known to be NP-complete (see, e.g., [15]).

Theorem 2. OpenDef is coNP-complete.

Proof. We first prove hardness. Fix an input graph $\boldsymbol{G}$ and a positive integer $k$. We may assume that $G$ is disjoint with the set of integers. Suppose first that $k=2 l$. Let $G^{\prime}$ be the graph with universe $G^{\prime}:=G \cup$ $\{-l, \ldots,-1,1, \ldots, l\}$ and with

$E^{G^{\prime}}:=E^{G} \cup\left\{\langle a, b\rangle \in\{-l, \ldots,-1,1, \ldots, l\}^{2}:|a-b|=1\right\} \cup\{\langle-1,1\rangle,\langle 1,-1\rangle\}$.

That is, $\boldsymbol{G}^{\prime}$ is the disjoint union of $\boldsymbol{G}$ and a path of length $k$. Define

$$
T:=\{\langle-l, \ldots,-1,1, \ldots, l\rangle,\langle l, \ldots, 1,-1, \ldots,-l\rangle\} .
$$

Now, observe that by Theorem 1 we have that OpenDef returns FALSE on input $\left(\boldsymbol{G}^{\prime}, T\right)$ if and only if InducedPath returns TRUE on input $(\boldsymbol{G}, k)$. The case where $k$ is odd is analogous. 
Showing that OpenDef is in coNP is a straightforward application of Theorem 1. Given a finite relational structure $\boldsymbol{A}$ and $T \subseteq A^{k}$, the fact that OpenDef returns FALSE on input $(\boldsymbol{A}, T)$ is witnessed by a bijection $\gamma$ between subsets of $A$ satisfying conditions easily checked in poly-time with respect to the size of $(\boldsymbol{A}, T)$.

Given a relational signature $\tau$ let OpenDef $[\tau]$ be the restriction of OpenDef to input structures of signature $\tau$. In view of the proof of Theorem 2 we have the following.

Corollary 3. OpenDef $\left[\tau_{\mathrm{GRAPH}}\right]$ is coNP-complete.

\section{Parameterized Complexity of OpenDef}

Parameterized complexity is a mathematical framework that allows for a more fine-grained analysis of the computational costs of a problem than classical complexity. In a parameterization of a (classical) problem we single out a specific part of the input of the problem to try and understand how this part affects the computational cost. For example, a parameterization of propositional SAT (i.e., the satisfiability problem for Propositional Logic) could be the number of variables in the input formula.

We begin with the basic definitions involved. There are slight discrepancies for these definitions in the literature, but our results remain valid regardless of which of the versions is used. We follow the account in [14]. As is customary, (classical) decision problems are formalized as languages over finite nonempty alphabets. Let $\Sigma \neq \emptyset$ be a finite alphabet.

- A parameterization of $\Sigma^{*}$ is a mapping $\kappa: \Sigma^{*} \rightarrow \mathbb{N}$ that is polynomial time computable.

- A parameterized (or parametric) problem (over $\Sigma$ ) is a pair $(Q, \kappa)$ consisting of a set $Q \subseteq \Sigma^{*}$ of strings over $\Sigma$ and a parameterization $\kappa$ of $\Sigma^{*}$.

We consider the following parameterization 2 of OpenDef:

\footnotetext{
${ }^{2}$ This parameterized problem (and others appearing below) is presented in an informal way, but it should be clear how to cast them in the form of our formal definition.
} 


\section{$p$-OpenDef Instance: A finite relational structure $\boldsymbol{A}$ and $T \subseteq$ $A^{m}$. \\ Parameter: $m|T|$. \\ Question: Is $T$ open-definable in $\boldsymbol{A}$ ?}

For a positive integer $k$, the $k$-th slice of a parameterized problem $(Q, \kappa)$ is the restriction of the problem to all instances $x \in \Sigma^{*}$ such that $\kappa(x)=k$. Let $p$-OpenDef ${ }_{k}$ denote the $k$-th slice of $p$-OpenDef.

Proposition 4. $p$-OpenDef ${ }_{k}$ is computable in time $k ! n^{2 k} p(n)$ where $n$ is the size of the input and $p(X)$ a polynomial.

Proof. Fix a finite relational structure $\boldsymbol{A}$ and $T \subseteq A^{m}$. Let $n$ be the size of $(\boldsymbol{A}, T)$ and $k=m|T|$. Given a positive integer $l$ let

$$
\operatorname{subIso}_{l} \boldsymbol{A}:=\{\gamma \in \text { subIso } \boldsymbol{A}:|\operatorname{dom} \gamma|=l\} \text {. }
$$

Note that Corollary 1 implies that $T$ is open-definable in $\boldsymbol{A}$ if and only if every $\gamma \in\left(\bigcup_{l \leq m} \operatorname{subIso}_{l} \boldsymbol{A}\right)$ preserves $T$. We show that the right-hand side of this equivalence can be checked in polynomial time. Let

$\mathcal{I}_{l}:=\left\{\gamma:\right.$ there are $B, B^{\prime} \subseteq A$ such that $|B|=l$ and $\gamma: B \rightarrow B^{\prime}$ is bijective $\}$.

Observe that

$$
\left|\mathcal{I}_{l}\right|=l ! \cdot\left(\begin{array}{c}
|A| \\
l
\end{array}\right)^{2}
$$

since there are $l$ ! bijections between any two subsets of size $l$ of $A$, and so

$$
\left|\mathcal{I}_{l}\right| \leq l ! \cdot|A|^{2 l} \leq k ! \cdot n^{2 k}
$$

Now for each $\gamma \in \mathcal{I}_{l}$ we have to check:

1. if $\gamma \in$ subIso $\boldsymbol{A}$,

2. and in that case, if $\gamma$ preserves $T$.

Clearly both these tasks can be carried out in time bounded by a polynomial in $n$. If $p_{l}(n)$ is such a polynomial, then checking if every member of 
subIso $_{l} \boldsymbol{A}$ preserves $T$ takes at most $\left|\mathcal{I}_{l}\right| \cdot p_{l}(n)$ steps. Thus, the computation for OpenDef ${ }_{k}$ on input $(\boldsymbol{A}, T)$ can be done in at most

$$
k ! n^{2 k} \sum_{l=1}^{k} p_{l}(n)
$$

steps.

A parameterized problem $(Q, \kappa)$ over the alphabet $\Sigma$ is fixed parameter tractable $(\mathrm{FPT})$ if there is an algorithm $\mathcal{A}$ together with a polynomial $p(X)$ and a computable function $f: \mathbb{N} \rightarrow \mathbb{N}$ such that $\mathcal{A}$ decides if $x \in Q$ in time $f(\kappa(x)) p(|x|)$ for a all $x \in \Sigma^{*}$. The class FPT of all fixed parameter tractable problems plays the role $\mathrm{P}$ plays in classical complexity.

Even though each slice of $p$-OpenDef can be computed in polynomial time, the bound given by Proposition 4 does not imply $p$-OpenDef $\in$ FPT, since the parameter appears as an exponent of the size of the input. In fact, as we shall see below it is unlikely that $p$-OpenDef is FPT, since it is hard for the class coW[1]; a class of parameterized problems believed to be strictly larger than FPT. But before we can discuss hardness of parameterized problems we need an adequate notion of reduction.

Definition 5. Let $(Q, \kappa)$ and $\left(Q^{\prime}, \kappa^{\prime}\right)$ be parameterized problems over the alphabets $\Sigma$ and $\Sigma^{*}$, respectively. An fpt-reduction from $(Q, \kappa)$ to $\left(Q^{\prime}, \kappa^{\prime}\right)$ is a mapping $R: \Sigma^{*} \rightarrow\left(\Sigma^{\prime}\right)^{*}$ such that:

1. For all $x \in \Sigma^{*}$ we have $\left(x \in Q \Leftrightarrow R(x) \in Q^{\prime}\right)$.

2. There is a computable function $f$ and a polynomial $p(X)$ such that $R(x)$ is computable in time $f(\kappa(x)) \cdot p(x)$.

3. There is a computable function $g: \mathbb{N} \rightarrow \mathbb{N}$ such that $\kappa(R(x)) \leq g(\kappa(x))$ for all $x \in \Sigma^{*}$.

If $P$ and $P^{\prime}$ are parameterized problems, we write $P \leq^{\text {fpt }} P^{\prime}$ if there is an fpt-reduction from $P$ to $P^{\prime}$, and write $P \equiv{ }^{\text {fpt }} P^{\prime}$ if there are fpt-reductions in both directions.

There are other notions of fpt-reduction, such as Turing fpt-reductions [14], involving oracles. All fpt-reductions in this note satisfy Definition 5 , and thus we simply use the name fpt-reduction for them. 
To analyze the complexity of parametric problems which appear not to be tractable, Downey and Fellows introduced the W hierarchy [8]. The classes

$$
\mathrm{FPT} \subseteq \mathrm{W}[1] \subseteq \mathrm{W}[2] \subseteq \cdots \subseteq \mathrm{W}[P]
$$

in this hierarchy are closed under fpt-reductions and are believed to be all different. They have many natural complete problems (see, e.g., [10, 14]). One can think of this hierarchy as analogous to the polynomial hierarchy in classical complexity. We only consider the classes $W[1]$ and $W[P]$ in the sequel. Their formal definitions are somewhat involved and not needed in our arguments, so we do not include them here. (The interested reader can find them in [14].) We do need the following characterization of $\mathrm{W}[P]$, analogous to the characterization of NP in terms of "certificates".

Lemma 6 ([14, Lem 3.8]). A parameterized problem $(Q, \kappa)$ over the alphabet $\Sigma$ is in $\mathrm{W}[P]$ if and only if there are computable functions $f, h: \mathbb{N} \rightarrow \mathbb{N}$, a polynomial $p(X)$, and a $Y \subseteq \Sigma^{*} \times\{0,1\}^{*}$ such that:

1. For all $(x, y) \in \Sigma^{*} \times\{0,1\}^{*}$ it is decidable in time $f(\kappa(x)) p(|x|)$ whether $(x, y) \in Y$.

2. For all $(x, y) \in \Sigma^{*} \times\{0,1\}^{*}$, if $(x, y) \in Y$ then $|y|=h(\kappa(x)) \log |x|$.

3. For every $x \in \Sigma^{*}$ we have $x \in Q$ iff there exists $y \in\{0,1\}^{*}$ such that $(x, y) \in Y$.

The complement $(Q, \kappa)^{\mathrm{C}}$ of a parametric problem $(Q, \kappa)$ is the parametric problem $\left(Q \backslash \Sigma^{*}, \kappa\right)$. It follows directly from the definitions that $P_{1} \leq^{\mathrm{fpt}} P_{2}$ iff $P_{1}^{\mathrm{C}} \leq{ }^{\mathrm{fpt}} P_{2}^{\mathrm{C}}$. For a class $\mathrm{K}$ of parametric problems, let coK denote the class off all parametric problems whose complement is in $\mathrm{K}$.

Proposition 7. $p$-OpenDef $\in \mathrm{coW}[P]$.

Proof. By Theorem 1 we know that $T \subseteq A^{m}$ fails to be open-definable in $\mathbf{A}$ if and only if there is a bijection $\gamma$ between subsets of $A$ of cardinality at most $m$, satisfying that $\gamma$ is a subisomorphism of $\mathbf{A}$ and does not preserve $T$. Such a bijection can be encoded as a binary string of length $O\left(m^{2} \log |A|\right)$, and we can compute in time polynomial in $\operatorname{size}(\mathbf{A}, T)$ if $\gamma$ is a subisomorphism of $\mathbf{A}$ not preserving $T$. (Note that this is a refinement of the argument we used in Theorem 2 to prove OpenDef $\in$ coNP.) 
Next we establish a lower bound for the complexity of $p$-OpenDef by a reduction from the following parameterized version of the Clique problem.

$$
\begin{aligned}
p \text {-Clique } & \text { Instance: } \\
\text { Parameter: } & k \text {. } \\
\text { Question: } & \text { Does } \boldsymbol{G} \text { have clique of size } k ?
\end{aligned}
$$

It is proved in [9, Cor 3.2] that $p$-Clique is complete (under fpt-reductions) for the class $\mathrm{W}[1]$.

Lemma 8. $p$-Clique $\leq^{f p t} p$-OpenDef ${ }^{C}$; hence $p$-OpenDef is hard for coW[1].

Proof. The idea is the same as in the proof of Theorem 2, Given an input $\mathbf{G}, k$ for $p$-Clique the reduction computes the input $\mathbf{G} \sqcup \mathbf{K}_{k}, T_{k}$ for $p$-OpenDef ${ }^{\mathrm{C}}$, where $\mathbf{G} \sqcup \mathbf{K}_{k}$ is the disjoint union of $\mathbf{G}$ with the complete graph on the vertices $\{1, \ldots, k\}$, and $T_{k}=\{(\sigma(1), \ldots, \sigma(k)): \sigma$ a permutation of $\{1, \ldots, k\}\}$. It is easy to see that this is an fpt-reduction, and that $\mathbf{G}$ has a clique of size $k$ iff $T_{k}$ is not open-definable in $\mathbf{G} \sqcup \mathbf{K}_{k}$. (Note that this is not a polynomial reduction.)

In contrast with our analysis of the classical complexity of OpenDef, we were not able to show that $p$-OpenDef is in coW[1]. However when we fix the vocabulary we can establish a sharp upper bound. For a vocabulary $\tau$ let $p$-OpenDef $[\tau]$ denote the restriction of $p$-OpenDef to input structures with vocabulary $\tau$, and let $p$-OpenDef ${ }^{C}[\tau]$ denote the complement of this problem. Before we start on the upper bounds, we have the following consequence of Theorem 8 ,

Corollary 9. For every vocabulary $\tau$ with at least one at least binary relation we have $p$-Clique $\leq{ }^{f p t} p$-OpenDef ${ }^{C}[\tau]$; and hence $p$-OpenDef $[\tau]$ is hard for $\mathrm{coW}[1]$.

Proof. If $\tau$ has at least one at least binary relation, it is easy to see that

$$
p \text {-OpenDef }{ }^{\mathrm{C}}\left[\tau_{\mathrm{GRAPH}}\right] \leq{ }^{\mathrm{fpt}} p \text {-OpenDef }{ }^{\mathrm{C}}[\tau] .
$$

From the proof of Lemma 8 it follows that $p$-Clique $\leq{ }^{\text {fpt }} p$-OpenDef ${ }^{\mathrm{C}}\left[\tau_{\text {GRAPH }}\right]$. 
We turn now to establishing an upper bound for $p$-OpenDef $[\tau]$. Recall that a sentence is existential if it has the form $\exists v_{1} \ldots \exists v_{l} \alpha\left(v_{1}, \ldots, v_{l}\right)$ where $\alpha$ is open. Let $\Sigma_{1}[\tau]$ be the set of all existential sentences over a vocabulary $\tau$. For a given vocabulary $\tau$, consider the following parameterized model checking problem:

$$
\begin{aligned}
p \text {-MC }\left(\Sigma_{1}[\tau]\right) \quad \text { Instance: } & \text { A finite } \tau \text {-structure } \mathbf{A} \text { and an existen- } \\
& \text { tial } \tau \text {-sentence } \varphi . \\
\text { Parameter: } & \operatorname{size}_{\tau}(\varphi) \\
\text { Question: } & \text { Does } \mathbf{A} \text { satisfy } \varphi \text { ? }
\end{aligned}
$$

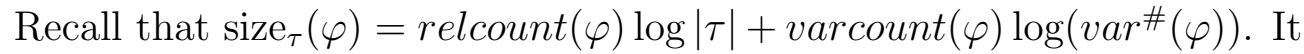
is proved in [13] that $p-\mathrm{MC}\left(\Sigma_{1}[\tau]\right)$ is in $\mathrm{W}[1]$ for all $\tau$.

Theorem 10. For every vocabulary $\tau$, $p$-OpenDef ${ }^{\mathrm{C}}[\tau] \leq{ }^{f p t} p-\mathrm{MC}\left(\Sigma_{1}[\tau]\right)$. Thus, $p$-OpenDef $[\tau] \in \operatorname{coW}[1]$.

Proof. Fix a $\tau$-structure $\mathbf{A}$ and $T \subseteq A^{m}$. For each tuple $\bar{a}=\left\langle a_{1}, \ldots, a_{m}\right\rangle \in$ $A^{m}$, and each $R \in \tau$ let $\Delta_{\bar{a}, R}\left(x_{1}, \ldots, x_{m}\right)$ be the the conjunction of the following set of atomic formulas

$$
\left\{R\left(x_{i_{1}}, \ldots, x_{i_{r}}\right):\left(a_{i_{1}}, \ldots, a_{i_{r}}\right) \in R^{\mathbf{A}}\right\} \cup\left\{\neg R\left(x_{i_{1}}, \ldots, x_{i_{r}}\right):\left(a_{i_{1}}, \ldots, a_{i_{r}}\right) \notin R^{\mathbf{A}}\right\},
$$

where $r$ is the arity of $R$. Observe that

$$
\begin{aligned}
\operatorname{size}\left(\Delta_{\bar{a}, R}\right) & =m^{r} \log |\tau|+r m^{r} \log m \\
& \leq q_{r}(m)
\end{aligned}
$$

for a suitable polynomial $q_{r}(X)$. Also observe that $\Delta_{\bar{a}, R}$ can be computed in time $O\left(m^{r} \operatorname{size}(\mathbf{A})+\operatorname{size}\left(\Delta_{\bar{a}, R}\right)\right)$, so there is a polynomial $p_{r}(X)$ such that the computation of $\Delta_{\bar{a}, R}$ can be done in at most $p_{r}(m) \operatorname{size}(\mathbf{A})$ steps. Next, define

$$
\Delta_{\bar{a}}\left(x_{1}, \ldots, x_{m}\right):=\bigwedge\left\{\Delta_{\bar{a}, R}\left(x_{1}, \ldots, x_{m}\right): R \in \tau\right\} .
$$

Let $\rho$ be the greatest among the arities of the relations in $\tau$. Then, $\operatorname{size}\left(\Delta_{\bar{a}}\right) \leq$ $|\tau| p_{\rho}(m)$, and $\Delta_{\bar{a}}$ is computable in time bounded by $|\tau| p_{\rho}(m) \operatorname{size}(\mathbf{A})$. Note that $\Delta_{\bar{a}}$ characterizes the isomorphism type of $\bar{a}$ in $\mathbf{A}$, i.e., for all $\bar{b} \in A^{m}$ we have

$$
\mathbf{A} \vDash \Delta_{\bar{a}}\left[b_{1}, \ldots, b_{m}\right] \Longleftrightarrow \bar{a} \mapsto \bar{b} \text { is a subisomorphism of } \mathbf{A} \text {. }
$$


Now, let

$$
\Delta_{T}\left(x_{1}, \ldots, x_{m}\right):=\bigvee\left\{\Delta_{\bar{a}}(\bar{x}): \bar{a} \in T\right\},
$$

and take $\varphi_{\mathbf{A}, T}$ as the sentence

$$
\exists \bar{x}_{1} \ldots \exists \bar{x}_{t+1} \bigwedge\left\{\bar{x}_{i} \neq \bar{x}_{j}: 1 \leq i<j \leq t+1\right\} \wedge \bigwedge\left\{\Delta_{T}\left(\bar{x}_{i}\right): 1 \leq i \leq t+1\right\}
$$

where $t$ is the number of tuples in $T$. It is straightforward to check that there are polynomials $p(X)$ and $q(X)$ such that:

(1) $\varphi_{\mathbf{A}, T}$ can be computed in time $p(m|T|) \operatorname{size}(\mathbf{A})$,

and

(2) $\operatorname{size}\left(\varphi_{\mathbf{A}, T}\right) \leq q(m|T|)$.

Next, note that $\mathbf{A} \vDash \Delta_{T}[\bar{b}]$ if and only if $\bar{b}$ has the same isomorphism type as some tuple in $T$; thus $\varphi_{\mathbf{A}, T}$ asserts that there are $t+1$ distinct $m$-tuples such that each one has the same isomorphism type as some tuple in $T$. So, $\mathbf{A} \vDash \varphi_{\mathbf{A}, T}$ if and only if there are $\bar{a} \in T$ and $\bar{b} \in A^{m} \backslash T$ such that $\bar{a} \mapsto \bar{b}$ is a subisomorphism of A. By Theorem 1, this says that:

(3) $\mathbf{A} \vDash \varphi_{\mathbf{A}, T}$ iff $T$ is not open-definable in $\mathbf{A}$.

To conclude, observe that (1-3) guarantee that the transformation $\mathbf{A}, T \rightsquigarrow$ $\mathbf{A}, \varphi_{\mathbf{A}, T}$ is an fpt-reduction from $p$-OpenDef ${ }^{C}[\tau]$ to $p-\mathrm{MC}\left(\Sigma_{1}[\tau]\right)$. (Notably, it is also a polynomial many-one reduction.)

It is worth to note that, by Corollary, Theorem 10 actually holds with $\equiv^{\mathrm{fpt}}$ in place of $\leq^{\mathrm{fpt}}$. That is,

$$
p \text {-OpenDef }{ }^{\mathrm{C}}[\tau] \equiv^{\mathrm{fpt}} p-\mathrm{MC}\left(\Sigma_{1}[\tau]\right)
$$

for every vocabulary $\tau$ with at least one at least binary relation.

Combining the upper an lower bounds found above we obtain the main result of this section.

Theorem 11. $p$-OpenDef $[\tau]$ is coW[1]-complete for every vocabulary $\tau$ with at least one at least binary relation. In particular, $p$-OpenDef $\left[\tau_{\mathrm{GRAPH}}\right]$ is coW[1]-complete.

Proof. Combine Corollary 9 and Theorem 10. 


\section{The Length of Open Formulas}

In previous sections we show that the definability problem for open formulas is not tractable. We now discuss the size of formulas required in definitions.

In particular, we show that it is not possible to polynomially bound the size of an open formula required in the definition of a relation in a given structure. More formally, we construct a sequence $\left\{\left(\mathbf{A}_{n}, T_{n}\right): n \in \mathbb{N}\right\}$ whose size grows polynomially in $n$, and such that the smallest definition of $T_{n}$ in $\mathbf{A}_{n}$ is exponentially large in $n$. This does not come as a surprise though; a polynomial bound on the size of defining open formulas would entail that OpenDef is in NP and, since we know OpenDef is coNP-complete (Theorem 2), we would have coNP $\subseteq \mathrm{NP}$.

Theorem 12. For each $n \geq 3$ there are a finite relational structure $\mathbf{A}_{n}$ and an $n^{2}$-ary relation $T_{n}$ over $A_{n}$ such that:

- $\operatorname{size}\left(\mathbf{A}_{n}, T_{n}\right)=O\left(n^{3} \log n\right)$,

- $T_{n}$ is open-definable in $\mathbf{A}_{n}$, and

- every open formula defining $T_{n}$ in $\mathbf{A}_{n}$ has at least $(n-1)^{n}$ literals.

Proof. Fix a natural number $n \geq 3$ and let $A$ be the union of four pairwise disjoint $n$-element sets, say

$$
A=\left\{a_{1}, \ldots, a_{n}\right\} \cup\left\{b_{1}, \ldots, b_{n}\right\} \cup\left\{c_{1}, \ldots, c_{n}\right\} \cup\left\{*_{1}, \ldots, *_{n}\right\} .
$$

Let $M_{1}$ be the $n \times n$ matrix such that

- the first column of $M_{1}$ is $\bar{a}$, and the rest of its entries are $*_{1}$.

For each $j \in\{2, \ldots, n\}$ take $M_{j}$ to be the $n \times n$ matrix such that

- the first column of $M_{j}$ is $\bar{b}$, the $j$-th column of $M_{j}$ is $\bar{c}$, and the rest of its entries are $*_{j}$.

Next, define

$$
\left.R:=\{\bar{a}, \bar{b}, \bar{c}\} \cup\left\{\text { rows of } M_{1}\right\} \cup\left\{\text { rows of } M_{2}\right\} \cup \cdots \cup \text { rows of } M_{n}\right\} \text {. }
$$


In what follows we identify $n^{2}$-tuples with $n \times n$ matrices (in the obvious way). We adjust the indexes of our variables accordingly, i.e., we shall write

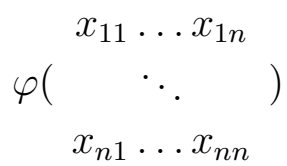

instead of $\varphi\left(x_{1}, \ldots, x_{n^{2}}\right)$.

Let $T=\left\{M_{1}\right\}$; we prove that $\mathbf{A}_{n}:=(A, R)$ and $T_{n}:=T$ satisfy the conditions of the theorem. First observe that, according to our definition of size (see Section 2.1), we have

$$
\begin{aligned}
\operatorname{size}\left(\mathbf{A}_{n}, T_{n}\right) & =\left(\operatorname{size}\left(\mathbf{A}_{n}\right)+n^{2}\right) \log 4 n \\
& =(1+n)+\left(4 n+n\left(3+n^{2}\right)+n^{2}\right) \log 4 n \\
& =O\left(n^{3} \log n\right)
\end{aligned}
$$

Next, define

$$
\alpha\left(\begin{array}{c}
x_{11} \ldots x_{1 n} \\
\ddots
\end{array}\right):=R\left(x_{11}, \ldots, x_{n 1}\right) \wedge \bigwedge_{j=1}^{n} R\left(x_{1 j}, \ldots, x_{n j}\right) .
$$

Suppose $M$ is an $n \times n$ matrix with entries in $A$ such that $\mathbf{A}_{n} \vDash \alpha(M)$. Then the first column of $M$, say $\bar{m}=\left(m_{1}, \ldots, m_{n}\right)$, and each of its rows must be in $R$. As each $m_{i}$ must be the first coordinate of some tuple in $R$, we have that $\bar{m} \in\{\bar{a}, \bar{b}\}$. If $\bar{m}=\bar{a}$, then $M=M_{1}$, since for each $i \in\{1, \ldots, n\}$ there is exactly one tuple in $R$ with first coordinate $a_{i}$. On the other hand, if $\bar{m}=\bar{b}$, we have that for each $i \in\{1, \ldots, n\}$ the $i$-th row of $M$ agrees with the $i$-th row of $M_{j_{i}}$ for some $j_{i} \in\{2, \ldots, n\}$.

Given $\bar{j} \in\{2, \ldots, n\}^{n}$ let $M^{\bar{j}}$ be the $n \times n$ matrix whose $i$-th row is the $i$-th row of $M_{j_{i}}$ for $i \in\{1, \ldots, n\}$. From the considerations in the last paragraph it follows that

$$
\left\{M: \mathbf{A}_{n} \vDash \alpha(M)\right\}=\left\{M_{1}\right\} \cup\left\{M^{\bar{j}}: \bar{j} \in\{2, \ldots, n\}^{n}\right\} .
$$

For each $\bar{j} \in\{2, \ldots, n\}^{n}$ define

$$
\lambda_{\bar{j}}\left(\begin{array}{c}
x_{11} \ldots x_{1 n} \\
\ddots \\
x_{n 1} \ldots x_{n n}
\end{array}\right):=R\left(x_{1 j_{1}}, \ldots, x_{n j_{n}}\right),
$$


and observe that $\mathbf{A}_{n} \vDash \lambda_{\bar{j}}\left(M^{\bar{j}}\right)$. So, if we take

$$
\beta\left(\begin{array}{c}
x_{11} \ldots x_{1 n} \\
x_{n 1} \ldots x_{n n}
\end{array}\right):=\bigwedge_{\bar{j} \in\{2, \ldots, n\}^{n}} \neg \lambda_{\bar{j}},
$$

it follows that $\mathbf{A}_{n} \vDash(\alpha \wedge \beta)(M) \Longleftrightarrow M=M_{1}$. Thus $T=\left\{M_{1}\right\}$ is opendefinable in $\mathbf{A}_{n}$.

To conclude, note that $\lambda_{\bar{j}}$ is the only atomic formula that distinguishes $M_{1}$ from $M^{\bar{j}}$. Thus, if $\varphi$ is an open formula that defines $T$, then $\neg \lambda_{\bar{j}}$ must occur in $\varphi$ for every $\bar{j} \in\{2, \ldots, n\}^{n}$.

\section{References}

[1] C. Areces, S. Figueira, and D. Gorín. Using logic in the generation of referring expressions. In S. Pogodalla and J. Prost, editors, Proceedings of the 6th International Conference on Logical Aspects of Computational Linguistics, volume 6736 of LNCS, pages 17-32. Springer, 2011.

[2] C. Areces, A. Koller, and K. Striegnitz. Referring expressions as formulas of description logic. In Proceedings of the Fifth International Natural Language Generation Conference, pages 42-49. ACL, 2008.

[3] M. Arenas and G. Diaz. The exact complexity of the first-order logic definability problem. ACM Transactions on Database Systems, 41(2):13:113:14, 2016.

[4] F. Bancilhon. On the completeness of query languages for relational data bases. In J. Winkowski, editor, Proceedings of the 7th Symposium of Mathematical Foundations of Computer Science, volume 64, pages 112-123. Springer, 1978.

[5] B. Berard, M. Bidoit, A. Finkel, F. Laroussinie, A. Petit, L. Petrucci, and P. Schnoebelen. Systems and Software Verification: Model-Checking Techniques and Tools. Springer, 2010.

[6] M. Campercholi and D. Vaggione. Semantical conditions for the definability of functions and relations. Algebra universalis, 76(1):71-98, 2016. 
[7] E. Clarke, O. Grumberg, and D. Peled. Model Checking. MIT Press, 1999.

[8] R. Downey and M. Fellows. Fixed-parameter tractability and completeness I: Basic results. SIAM Journal on Computing, 24(4):873-921, 1995.

[9] R. Downey and M. Fellows. Fixed-parameter tractability and completeness II: On completeness for W[1]. Theoretical Computer Science, 141(1):109-131, 1995.

[10] R. Downey and M. Fellows. Fundamentals of Parameterized Complexity. Springer Publishing Company, Incorporated, 2013.

[11] H. Ebbinghaus, J. Flum, and W. Thomas. Mathematical Logic. SpringerVerlag, 1994.

[12] S. Figueira and D. Gorín. On the size of shortest modal descriptions. In L. Beklemishev, V. Goranko, and V. Shehtman, editors, Advances in Modal Logic, volume 8, pages 114-132. College Publications, 2010.

[13] J. Flum and M. Grohe. Fixed-parameter tractability, definability, and model-checking. SIAM Journal on Computing, 31(1):113-145, 2001.

[14] J. Flum and M. Grohe. Parameterized Complexity Theory. Texts in Theoretical Computer Science. An EATCS Series. Springer-Verlag New York, Inc., Secaucus, NJ, USA, 2006.

[15] M. Garey and D. Johnson. Computers and Intractability: A Guide to the Theory of NP-Completeness. W. H. Freeman and Co., 1979.

[16] P. Jeavons, D. Cohen, and M. Gyssens. How to determine the expressive power of constraints. Constraints, 4(2):113-131, 1999.

[17] E. Krahmer and K. van Deemter. Computational generation of referring expressions: A survey. Computational Linguistics, 38(1):173-218, 2012.

[18] J. Paredaens. On the expressive power of the relational algebra. Information Processing Letters, 7(2):107-111, 1978.

[19] R. Willard. Testing expressibility is hard. In Proceedings of the 16th International Conference on Principles and Practice of Constraint Programming, pages 9-23, 2010. 